\title{
USP39 promotes the growth of human hepatocellular carcinoma in vitro and in vivo
}

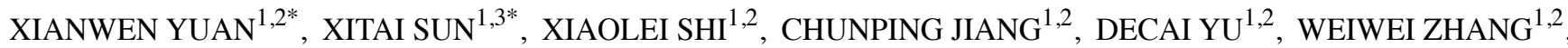

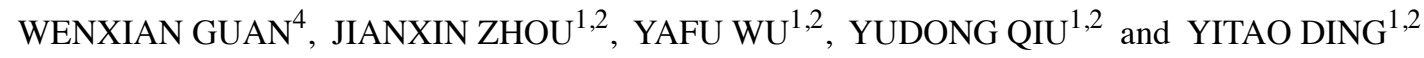 \\ ${ }^{1}$ Department of Hepatobiliary Surgery, The Affiliated Drum Tower Hospital of Nanjing University Medical School; \\ ${ }^{2}$ Institute of Hepatobiliary Surgery of Nanjing University; ${ }^{3}$ Center of Minimally Invasive Surgery, and \\ ${ }^{4}$ Department of General Surgery, The Affiliated Drum Tower Hospital of Nanjing University \\ Medical School, Nanjing, Jiangsu 210008, P.R. China
}

Received April 7, 2015; Accepted June 2, 2015

DOI: 10.3892/or.2015.4065

\begin{abstract}
Ubiquitin specific protease 39 (USP39) plays an important role in mRNA splicing. In the present study, we investigated the role of USP39 in regulating the growth of hepatocellular carcinoma (HCC). We detected USP39 expression in more than 100 HCC clinical samples. The USP39 expression was significantly higher in the tumor tissues compared to the adjacent normal tissues, and was strongly associated with the pathological grade of HCC. USP39 knockdown inhibited cell proliferation and colony formation in vitro in the HepG2 cells, while upregulation of USP39 promoted tumor cell growth. FCM assay showed that USP39 knockdown led to G2/M arrest and induced apoptosis in the HepG2 cells. USP39 knockdown by shRNA inhibited xenograft tumor growth in nude mice. Moreover, USP39 knockdown led to the upregulation of p-Cdc2 and downregulation of $\mathrm{p}-\mathrm{Cdc} 25 \mathrm{c}$ and $\mathrm{p}$-myt1, while the expression of total Cdc2, Cdc25c and myt1 was not changed in the USP39knockdown cells. We also found that p-Cdc2 was decreased in the USP39-overexpressing cells and was upregulated in the xenografted tumors derived from the HepG2/KD cells from nude mice. Meanwhile, the expression levels of FoxM1 and its target genes PLK1 and cyclin B1 were decreased in the USP39-knockdown cells. These results suggest that USP39 may contribute to FoxM1 splicing in HCC tumor cells. Our data indicate that USP39 knockdown inhibited the growth of $\mathrm{HCC}$ both in vitro and in vivo through $\mathrm{G} 2 / \mathrm{M}$ arrest, which was partly achieved via the inhibition of FoxM1 splicing.
\end{abstract}

Correspondence to: Professor Yitao Ding, Department of Hepatobiliary Surgery, The Affiliated Drum Tower Hospital of Nanjing University Medical School, 321 Zhongshan Road, Nanjing, Jiangsu 210008, P.R. China

E-mail: dytnanjing1983@126.com

*Contributed equally

Key words: hepatocellular carcinoma, USP39, G2/M arrest, FoxM1

\section{Introduction}

Hepatocellular carcinoma (HCC) is one of the most common cancers, and accounts for 600,000 deaths annually (1). Recently, the discovery of new targets in the molecular pathways in HCC has achieved significant results (2).

The growth of tumor cells is regulated by various proteins called cyclin-dependent protein kinases (Cdks) that consist of a family of heterodimeric serine/threonine kinases (3). The cell cycle consists of DNA synthesis (S) and mitotic (M) phases separated by gap phases in the order G1-S-G2-M (4). DNA damage at the $\mathrm{G} 2 / \mathrm{M}$ checkpoint prevents cells from entering mitosis (M-phase). The activity of the cyclin B-Cdc2 complex is pivotal in regulating G2-phase transition (5).

The Forkhead box protein M1 (FoxM1) is a member of the forkhead transcription factor family, which has been shown to have an important role in controlling the cell cycle. In particular, FoxM1 controls mitotic entry through the periodic upregulation of a group of genes that are maximally expressed as cells progress through late $\mathrm{G} 2$ and into $\mathrm{M}$ phase $(6,7)$. The FoxM1 transcript undergoes alternative splicing to produce three alternative isoforms (8).

There are a number of target genes of FoxM1 implicated in mitosis, such as cyclin B, Aurora B kinase, CENPA and PLK1 $(9,10)$. Cyclin B is an important gene in $\mathrm{G} 2 / \mathrm{M}$ transition. Aurora B kinase is a protein that functions in the attachment of the mitotic spindle to the centromere. Centromere protein A (CENPA) is a protein that encodes centromere protein. Polo-like kinase 1 (PLK1) belongs to a family of serine-threonine kinases and plays a critical role in mitotic progression. Human Cdc25 genes consist of three isoforms: Cdc25A, B and C $(11,12)$. In mammalian cells, all three Cdc25 isoforms are associated with cell cycle regulation because of their functions in the dephosphorylation of Cdk1 and Cdk2. Cdc25A regulates both G1/S and S/G2 transition (13), while $\mathrm{Cdc} 25 \mathrm{~B}$ and $\mathrm{Cdc} 25 \mathrm{C}$ regulate late $\mathrm{G} 2$ and mitosis, respectively (14-16). Moreover, $\mathrm{Cdc} 25 \mathrm{C}$ is an upstream factor of Cdc2 and can be phosphorylated by PLK1 (17).

Deubiquitinating enzymes (DUBs) are proteases that regulate ubiquitin or ubiquitin-like gene products. The human genome encodes nearly 100 DUBs with specificity 
for ubiquitin in 5 families (18). Ubiquitin specific protease 39 (USP39) is one of the DUBs without ubiquitin protease activity, as it lacks three important residues for protease activity in DUBs (19). USP39 encodes a conserved protein of the U4/U6.U5 tri-snRNP, with $65 \%$ overall homology to the yeast Sad1p splice factor, and it has been implicated in the assembly of mature spliceosome. In vitro, USP39 appears to be essential for pre-mRNA splicing, but not for the stability of the spliceosome complex once it is formed (20). USP39 is also essential in the spindle assembly checkpoint and regulates the Aurora B mRNA levels in U2OS cells (19). In addition, zebrafish USP39 mutation can induce G1/S arrest by rb1 splicing defect, and the e2f4 is a target of USP39. USP39 mutation contributes to adenohypophyseal sensitivity to rb1 and e2f4 that causes pituitary tumorigenesis (20). USP39 may act as an oncogenic factor in breast cancer, and downregulation of USP39 was found to induce the apoptosis of MCF-7 breast cancer cells (21). Thus, USP39 may be a potential molecular target for cancer therapy. However, the roles of USP39 in HCC have been rarely studied. In the present study, we aimed to investigate the potential roles of USP39 in hepatocellular cancer. We found that the expression levels of USP39 were higher in tumor tissues than in adjacent normal tissues, and the expression of USP39 was strongly associated with the pathological grade. Thus, we hypothesized that USP39 may act as a new target in liver cancer. To verify our hypothesis, we stably ablated USP39 expression by shRNA in hepatocellular carcinoma cell line HepG2 and investigated the changes in cell proliferation in vitro and tumor growth in vivo. We demonstrated that inhibition of USP39 suppressed the tumorigenesis of HCC via the inhibition of FoxM1 splicing.

\section{Materials and methods}

Tissue array and evaluation of immunostaining. HCC tissue arrays were purchased from the National Engineering Center for Biochips (Shanghai, China). The expression of USP39 in the tissues was evaluated by immunohistochemical staining using the USP39-specific antibody. The staining was scored according to the staining intensity and the percentage of positive cells, and the final staining scores were calculated as the score of the staining intensity multiplied by the score of the percentage of positive cells.

Cell growth assay. Cell growth was measured by multiparametric high-content screening (HCS). HepG2 cells were infected with either the NC lentivirus or the USP39 siRNA lentivirus and were seeded at 2,000 cells/well in 96-well plates, and then incubated at $37^{\circ} \mathrm{C}$ with $5 \% \mathrm{CO}_{2}$ for 5 days. The plates were processed with ArrayScan ${ }^{\mathrm{TM}}$ HCS software (Cellomics Inc.) and kept at $4^{\circ} \mathrm{C}$ for up to $24 \mathrm{~h}$ before each day's analysis. The system can identify stained cells and report the intensity and distribution of fluorescence in each individual cell. In each well, at least 800 cells were analyzed. Images and data were stored in a Microsoft SQL database for easy retrieval.

Cell culture and MTT assay. Human HCC cell lines, HepG2, SMMC-7721, BEL-7402 and Huh-7, were purchased from the Shanghai Institutes for Biological Sciences (China). All cell lines were cultured in RPMI-1640 medium supplemented with
$10 \%$ fetal bovine serum (FBS), $100 \mathrm{U} / \mathrm{ml}$ of penicillin and $100 \mu \mathrm{g} / \mathrm{ml}$ of streptomycin (complete medium) and maintained at $37^{\circ} \mathrm{C}$ with $5 \% \mathrm{CO}_{2}$.

HCC cells ( $1 \times 10^{5}$ in $0.2 \mathrm{ml} /$ well) were seeded in a 96 -well plate in three replicates, and cultured in complete medium at $37^{\circ} \mathrm{C}$ for $1-8$ days. One hundred microliters of MTT solution $(5 \mathrm{mg} / \mathrm{ml})$ was added into each well and incubated at $37^{\circ} \mathrm{C}$ for $4 \mathrm{~h}$. The supernatant was then removed and $150 \mu \mathrm{l}$ of DMSO was added per well. The plate was oscillated for $30 \mathrm{~min}$ at room temperature. Absorbance at $490 \mathrm{~nm}$ was measured and the values were determined after background subtraction. The experiment was repeated at least three times.

Vector construction and transfection. The siRNA targeting the USP39 sequences (KD, ACCAAGTTGCCTCCATATCTA and KD\#, CCAGACAACTATGAGATCATCGATT) and the non-silencing sequence (TTCTCCGAACGTGTCACGT) were transformed into short hairpin RNA (shRNA) (stem-loop-stem structure) and cloned into the pGCSIL-GFP lentiviral vector (GeneChem Co., Ltd., Shanghai, China) after AgeI/EcoRI digestion. The recombinant plasmid and two virus packaging plasmids (GeneChem) were transfected into the human 293T cell line using Lipofectamine 3000 (Invitrogen Life Technologies, Grand Island, NY, USA) following the manufacturer's instructions. After 3 days of incubation, the lentivirus from the culture medium was collected. For stable infection, the HCC cell lines were cultured in 6-well plates, and infected with the USP39 shRNA-expressing lentivirus (USP39-shRNA) or the non-silencing shRNA-expressing lentivirus (control) with a multiplicity of infection (MOI) of 10. Five days after infection, the cells were observed under fluorescence microscopy (DMI4000B; Leica Microsystems, Germany). For USP39 overexpression, full length of USP39 cDNA was amplified by PCR and cloned into pEGFP-N2 (BD, Franklin Lakes, NJ, USA). The recombinant plasmids were transfected into cells using Lipofectamine 3000 (Invitrogen) following the manufacturer's instructions.

Tumor xenografts. BALB/c nude mice aged 4-6 weeks were obtained from the Laboratory Animal Centre of the Affiliated Drum Tower Hospital of Nanjing University Medical School and maintained in a standard pathogen-free condition. All experiments were approved by the Institutional Animal Care and Use Committee of the Affiliated Drum Tower Hospital of Nanjing University Medical School. Tumor cells $\left(2 \times 10^{6}\right)$ in $0.2 \mathrm{ml}$ serum-free RPMI-1640 medium were subcutaneously injected into the flank of each mouse. The right flank was injected with tumor cells containing USP39 shRNA and control tumor cells were injected into the left flank of each mouse. Each group consisted of 10 mice. Tumor growth was monitored by measuring the length (L) and width (W) of each tumor using a caliper, and tumor size was calculated by the formula $\mathrm{L} \times \mathrm{W}^{2} \times(\pi / 6)$. At the end of the experiment, the tumors were isolated from the mice and fixed in $4 \%$ paraformaldehyde and paraffin-embedded.

Quantitative real-time PCR ( $q R T-P C R)$. Total RNA was extracted from the HCC cell lines using TRIzol (Invitrogen) following the manufacturer's instructions. qRT-PCR was performed as reported previously (22). Briefly, $1 \mu \mathrm{g}$ of total 
Table I. Correlation of the expression of USP39 with clinicopathological features in the HCC tissue array.

\begin{tabular}{|c|c|c|c|c|c|c|c|c|c|}
\hline \multirow[b]{2}{*}{ Characteristics } & \multirow[b]{2}{*}{$\mathrm{N}$} & \multicolumn{5}{|c|}{ USP39 expression } & \multirow[b]{2}{*}{ Mean rank } & \multirow[b]{2}{*}{$\mathrm{Z}$} & \multirow[b]{2}{*}{ P-value } \\
\hline & & 0 & 1 & 2 & 3 & None & & & \\
\hline \multicolumn{10}{|l|}{ Gender } \\
\hline Male & 104 & 2 & 14 & 5 & 81 & 2 & 61.14 & -1.204 & 0.229 \\
\hline Female & 18 & 1 & 2 & 3 & 11 & 1 & 53.18 & & \\
\hline \multicolumn{10}{|l|}{ Age (years) } \\
\hline$<50$ & 49 & 2 & 9 & 6 & 31 & 1 & 52.65 & -2.614 & 0.009 \\
\hline$\geq 50$ & 73 & 1 & 7 & 2 & 61 & 2 & 64.97 & & \\
\hline \multicolumn{10}{|l|}{ Tumor size $(\mathrm{cm})$} \\
\hline$<5$ & 67 & 1 & 8 & 4 & 52 & 2 & 59.97 & -0.729 & 0.466 \\
\hline$\geq 5$ & 52 & 2 & 7 & 4 & 38 & 1 & 56.63 & & \\
\hline \multicolumn{10}{|l|}{ Differentiation } \\
\hline 1 & 6 & 0 & 3 & 0 & 3 & 0 & 42.50 & 9.219 & 0.027 \\
\hline 2 & 75 & 3 & 11 & 7 & 53 & 1 & 56.66 & & \\
\hline 3 & 40 & 0 & 2 & 1 & 35 & 2 & 68.92 & & \\
\hline 4 & 1 & 0 & 0 & 0 & 1 & 0 & 73.5 & & \\
\hline \multicolumn{10}{|l|}{ TNM stage } \\
\hline I & 23 & 0 & 2 & 0 & 20 & 1 & 38.64 & 1.703 & 0.636 \\
\hline II & 26 & 1 & 3 & 1 & 21 & 0 & 34.90 & & \\
\hline III & 22 & 0 & 2 & 1 & 18 & 1 & 36.93 & & \\
\hline IV & 4 & 0 & 0 & 0 & 4 & 0 & 42.00 & & \\
\hline \multicolumn{10}{|l|}{ Location } \\
\hline Tumor tissue & 122 & 3 & 16 & 8 & 92 & 3 & & -7.499 & 0.000 \\
\hline Adjacent tissue & 118 & 43 & 37 & 6 & 31 & 1 & & & \\
\hline
\end{tabular}

A tissue microarray was stained with anti-human USP39 antibody (1:150, ab131244; Abcam). The staining intensity was scored on a scale of 5 as follows: negative $(0+)$, weak (1+), moderate $(2+)$ and strong $(3+)$. None means that the site is lost in the section. HCC, hepatocellular carcinoma.

RNA was transcribed using random primers and Primescript reverse transcriptase (Takara, Dalian, China). Quantitative PCR reaction for the indicated genes was carried out using SYBR-Green qPCR kit (Takara) on a fluorescent temperature cycler (Mx3000P Real-Time PCR system; Stratagene, La Jolla, CA, USA). The following primers were used to detect the expression of USP39 (forward, 5'-CCAGCGATGGCAAC TAC-3' and reverse, 5'-ACCACAACGGAAACACG-3'); and GAPDH (forward 5'-TGACTTCAACAGCGACACCCA-3' and reverse, 5'-CACCCTGTTGCTGTAGCCAAA-3').

PCR reaction was performed with the parameters of denaturation: $95^{\circ} \mathrm{C}$ for $5 \mathrm{~min}$, followed by 45 cycles of $95^{\circ} \mathrm{C}$ for $15 \mathrm{sec}$ and $60^{\circ} \mathrm{C}$ for $1 \mathrm{~min}$. Using GAPDH as an endogenous control, relative gene expression was determined by the comparative $\mathrm{Ct}$ method. The melting curve of a product is sequence-specific and can be used to distinguish nonspecific from specific PCR products. Gene expression was analyzed with the Stratagene analysis software. The experiment was repeated at least three times.

Flow cytometric analysis. Cells transfected with the lentivirus were harvested, washed twice with cold PBS, fixed with cold $70 \%$ ethanol overnight, and resuspended with PBS. The suspension was filtrated through a 400-mesh membrane. The cells were stained with propidium iodide (PI) or Annexin V-APC (eBioscience, Inc.), and then analyzed using a BD FACSCalibur flow cytometer (BD Biosciences, San Diego, CA, USA). The experiment was repeated at least three times.

Western blotting. Total proteins were extracted from the tumor cell lines in RIPA buffer containing fresh protease and phosphatase inhibitors. The protein concentration was determined using the BCA assay (Pierce, Rockford, IL, USA). Twenty micrograms of protein was separated on $10 \%$ SDS-PAGE and transferred onto a PVDF membrane. The membrane was blocked with $3 \% \mathrm{BSA}$ in $10 \mathrm{mM}$ Tris- $\mathrm{HCl}$ (pH 7.4) containing $0.05 \%$ Tween-20 and incubated with a primary antibody at $4^{\circ} \mathrm{C}$ for $12 \mathrm{~h}$. After washing with Tris- $\mathrm{HCl}$ buffer for 3 times, the membrane was incubated with a corresponding peroxidase-conjugated secondary antibody (Santa Cruz Biotechnology, Santa Cruz, CA, USA), and developed in SuperSignal West Pico Chemiluminescent Substrate (Pierce). The protein was visualized by autoradiography and quantified by densitometric analysis using a Versadoc Imaging System Model 3000. The experiment was repeated at least three times.

Statistical analyses. All statistical analyses were performed using SPSS 11.0 software (SPSS Inc., Chicago, IL, USA). 


\section{A}
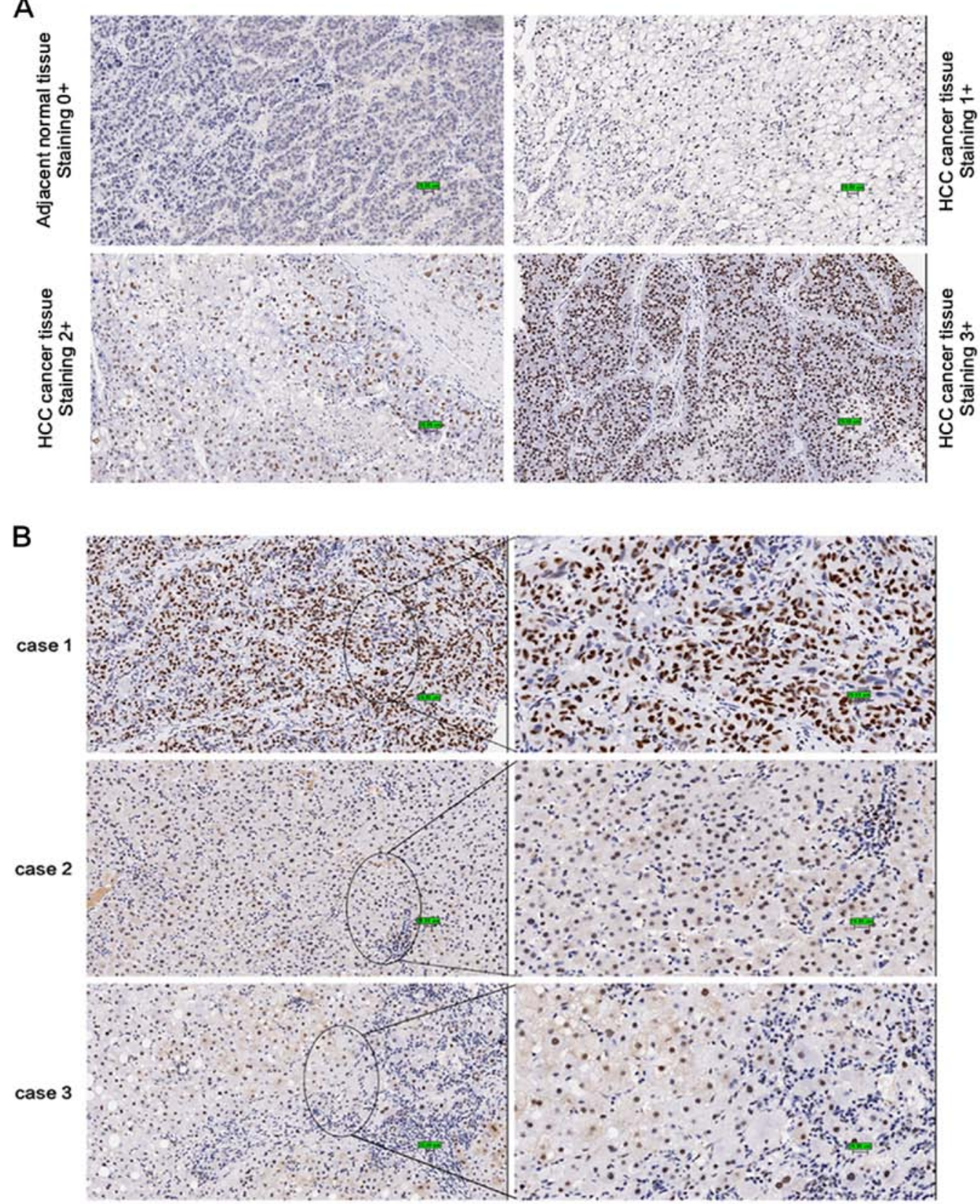

Figure 1. Immunohistochemical analyses of USP39 expression in human tissue microarrays. (A) Representative staining of USP39 expression in tumor tissues and corresponding adjacent normal tissues. Normal liver tissues showed weak/undetectable USP39 staining (score 0 or 1+), while HCC tissues showed high (score 3+), moderate (score 2+) or low (score 1+) USP39 staining. (B) Representive immunostaining in tumor tissues with different pathological grades and adjacent normal tissues. USP39 was highly expressed in tumor cells but weakly in normal liver cells (case 1 and 2). The expression of USP39 was much higher in well-differentiated HCC cells (case 1) than in poorly differentiated HCC cells (case 3). Left panels: magnification, x100; right panels: magnification, x200. HCC, hepatocellular carcinoma.

Differences among categorical variables were analyzed using one-way ANOVA/SNK test or independent-sample Student's t-test. The xenografted tumors were analyzed with the paired t-test. The immunoreactive scores for USP39 for tissue array were analyzed using non-parametric Mann-Whitney U, Kruskal-Wallis $\mathrm{H}$ and Wilcoxon tests. $\mathrm{P}<0.05$ was considered statistically significant.

\section{Results}

Expression of USP39 in the clinical HCC tissues. Using a USP39-specific antibody, we analyzed the USP39 protein level and distribution in the HCC tissue array by immunohistochemical staining. The array included 116 HCC patient samples including cancer and corresponding adjacent normal tissues, 6 cancer tissue samples and 2 adjacent normal tissue samples. The HCC patients included 104 males and 18 females, with a median age of 52 years (range, 14-73 years). Only 75 patients in the array had TNM staging. As shown in Table I and Fig. 1A, USP39 was strongly expressed in the HCC tumor tissues; 103 of the 122 cancer tissues had a score of $\geq 2$, while USP39 expression in the adjacent normal tissues was markedly decreased compared with that in the cancer tissues $(\mathrm{Z}=-7.499, \mathrm{P}<0.001)$. As shown in Fig. 1B, USP39 was 


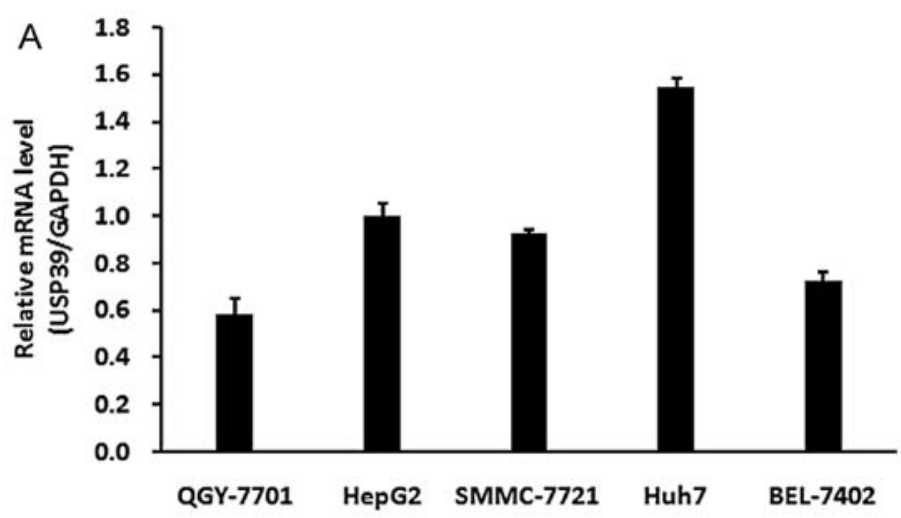

B

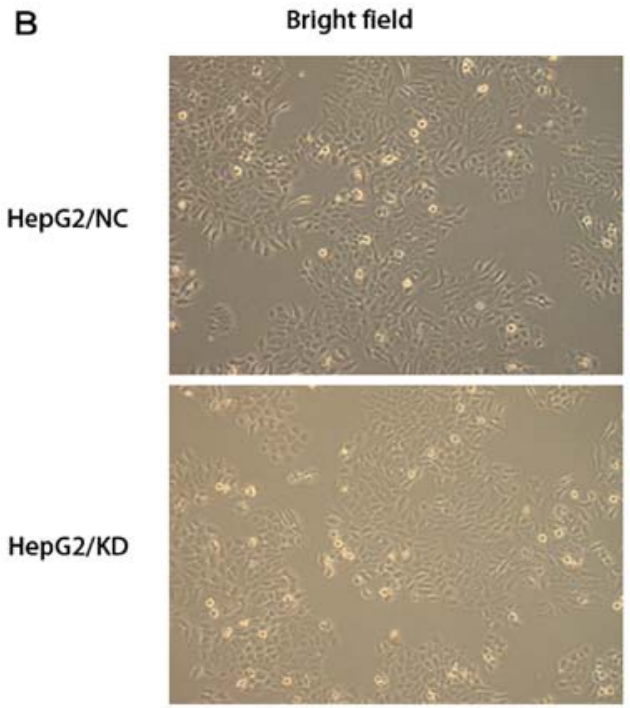

Fluorescence field
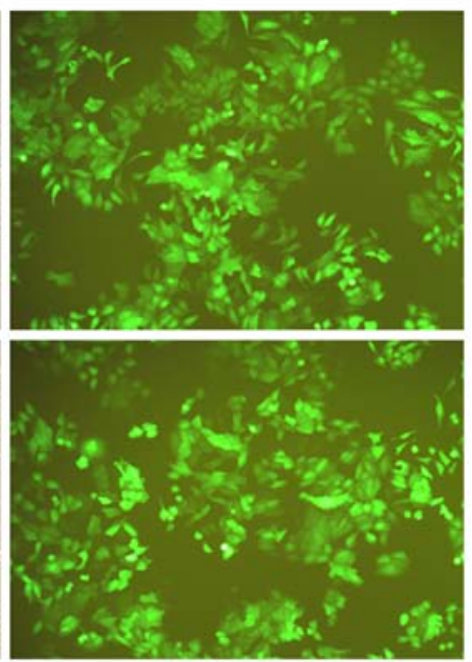

Figure 2. Detection of USP39 in HCC cell lines and transfection of USP39-lentiviral knockdown. (A) The mRNA levels of USP39 in HCC cell lines: QGY-7701, SMMC-7721, HepG2, Huh7 and BEL-7402. USP39 expression was extremely high in Huh7, high in HepG2, SMMC-7721 and BEL-7402, and moderate in QGY-7701. (B) Lentiviral infection. Representative GFP expression in HepG2/NC cells (upper panels) and HepG2/KD cells (lower panel). Left panels, bright field; right panel, fluorescence field. Magnification, x100. HCC, hepatocellular carcinoma.

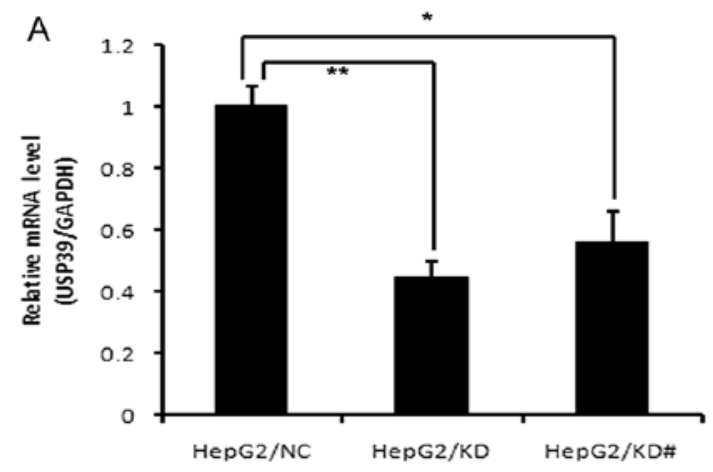

B

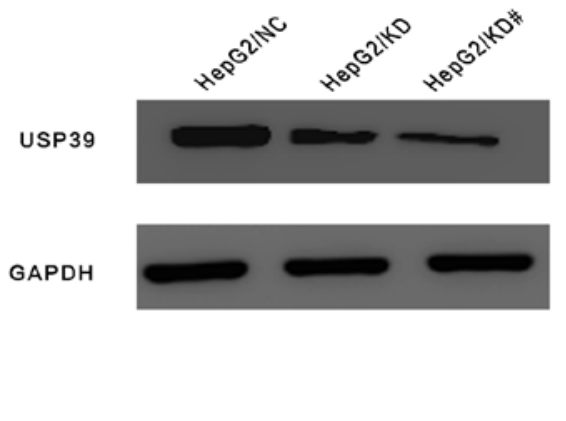

C

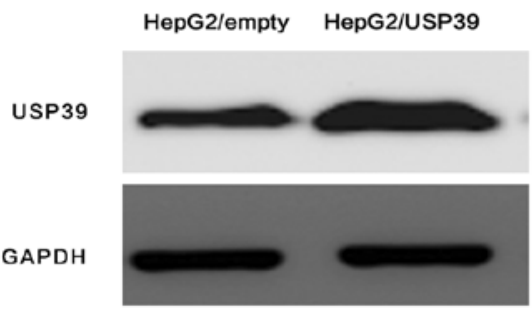

Figure 3. Efficiency of the knockdown and overexpression of USP39 in the HepG2 cells. (A) The expression of USP39 mRNA in the HepG2/NC, HepG2/ KD and HepG2/KD\# cells was examined by real-time PCR. (B) The expression of USP39 protein in the HepG2/NC, HepG2/KD and HepG2/KD\# cells was examined by western blotting. (C) Overexpression efficiency of USP39 in the HepG2 cells was detected by western blotting, and GAPDH was used as an internal control. $\left({ }^{*} \mathrm{p}<0.05,{ }^{* *} \mathrm{p}<0.01\right)$. 
A
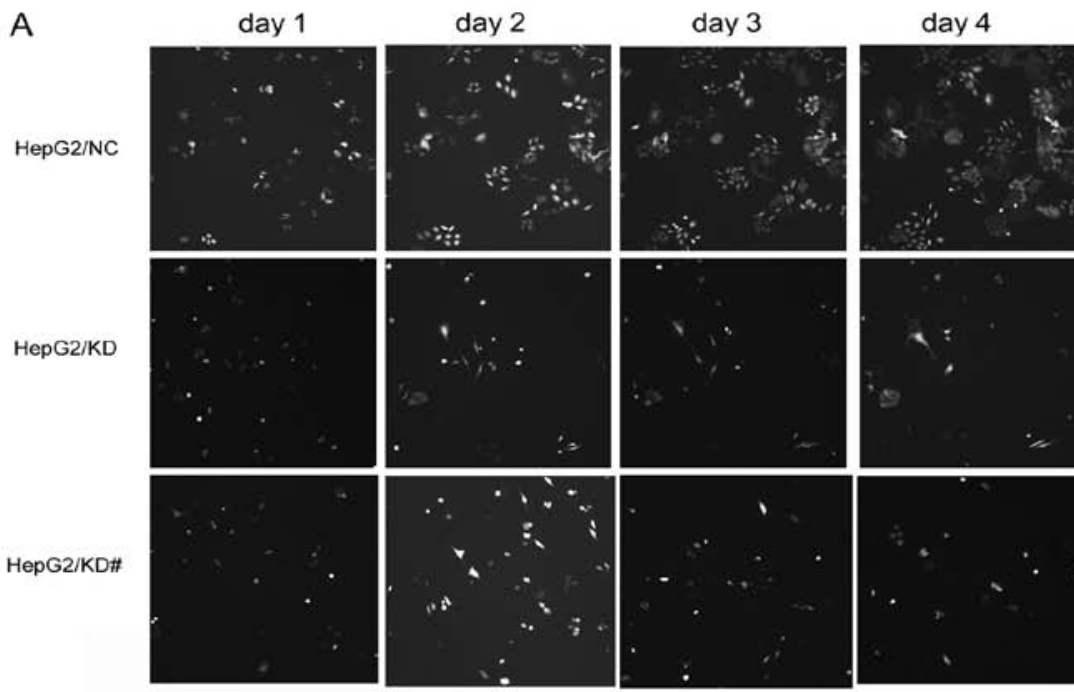

day 5
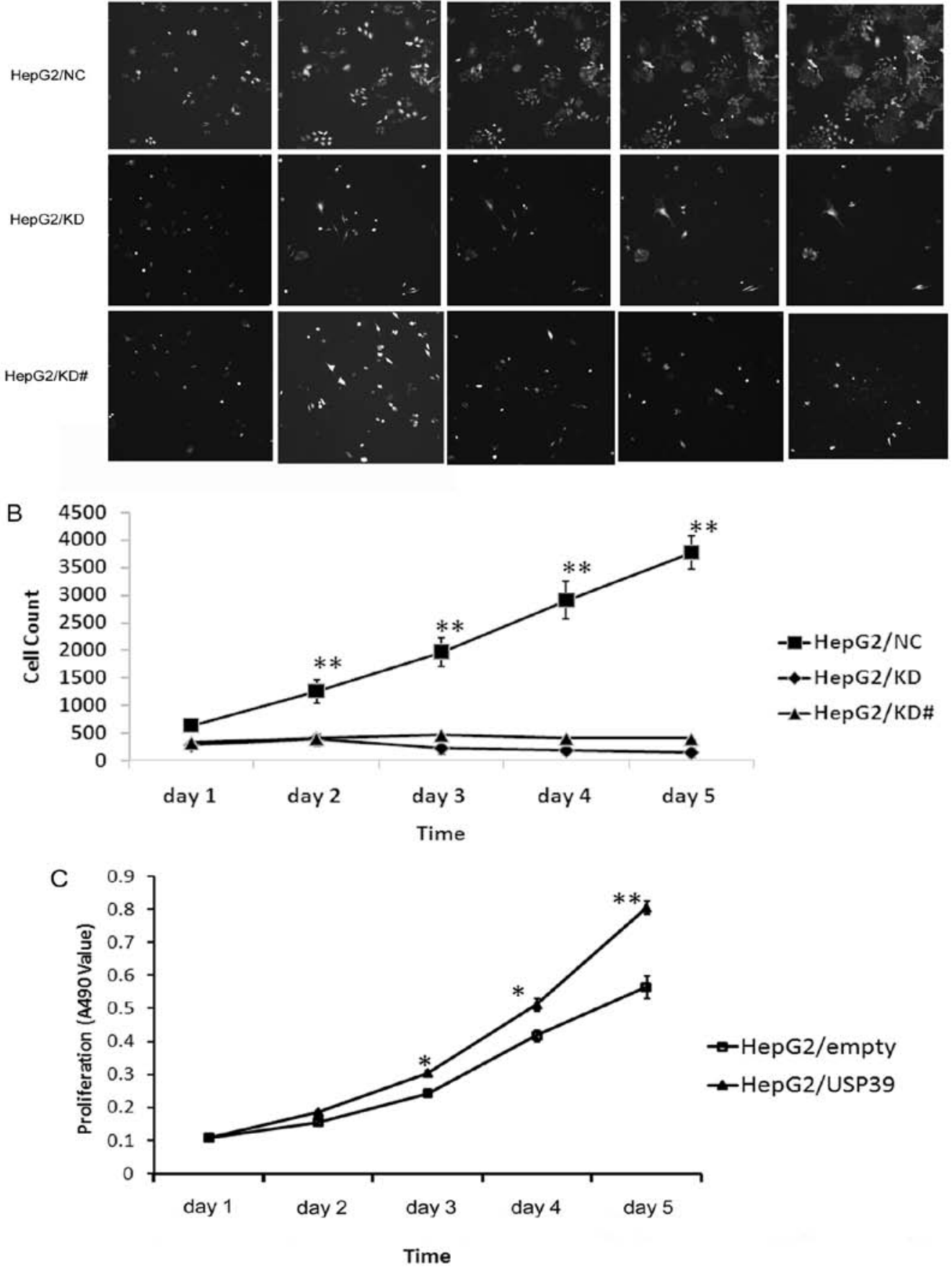

Figure 4. Effect of the downregulation of USP39 on HepG2 cell growth. (A) USP39-siRNA targeted cells and negative control cells were observed by Cellomics for 5 days. (B) Cell count curve of the USP39-siRNA targeted cells and negative control cells. The cell proliferation ability was significantly inhibited in the $\mathrm{HepG} 2 / \mathrm{KD}$ and HepG2/KD\# cells as compared with that in the negative control cells. (C) MTT assay was used to assess the cell proliferation in the HepG2/ empty and HepG2/USP39 cells. Overexpression of USP39 significantly enhanced the growth of the HepG2 cells ( $\left.{ }^{*} \mathrm{p}<0.05,{ }^{* *} \mathrm{p}<0.01\right)$.

highly expressed in the tumor cells but weakly expressed in the normal liver cells (case 1 and 2). Notably, the expression of USP39 was associated with the pathological grade of HCC ( $\mathrm{Z}=9.219, \mathrm{P}<0.05$; Table I); well-differentiated tumor cells showed a high level of expression (case 1) while poorly differentiated tumor cells exhibited a low level of expression (case 3). Our results also indicated that the expression of USP39 was higher in older individuals (age $\geq 50$ ) than that in younger individuals (age $<50$ ). Yet, USP39 expression did not appear to be associated with gender, tumor size and TNM stage. Thus, we can draw a conclusion that the expression of USP39 may be associated with the tumorigenesis of HCC (Fig. 1).

Expression of USP39 in HCC cell lines. We examined the level of USP39 expression in 5 HCC cell lines including HepG2, SMMC-7721, BEL-7402, QGY-7701 and Huh7 using qRT-PCR. High expression of USP39 mRNA was observed in all 5 HCC cell lines (Fig. 2A). 

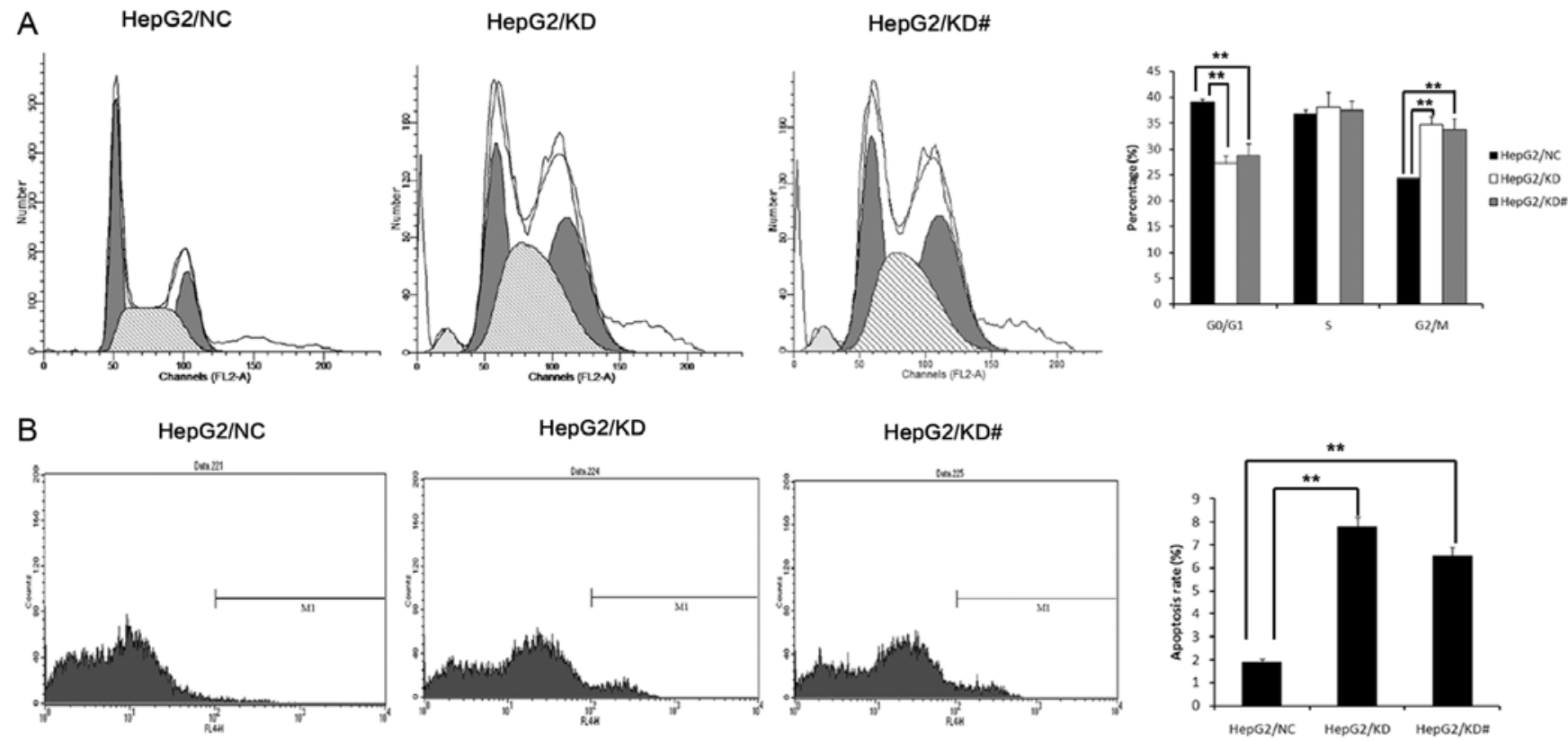

Figure 5. USP39 knockdown inhibits the growth of HepG2 cells in vitro. Flow cytometric analysis was used to analyze the cell cycle distribution in the HepG2 cells after viral infection for 5 days. The percentage of cells in the G2/M phase was markedly increased (A) and the apoptosis rate was significantly enhanced (B) after USP39 knockdown $\left(^{* *} \mathrm{p}<0.01\right)$.

Effects of USP39 on the proliferation and colony formation of HCC cells. To verify the roles of USP39 in the tumorigenesis of HCC in vitro, we inhibited USP39 expression in the HCC cell lines by shRNA targeting USP39 (Fig. 2B). USP39 expression was significantly decreased in the HepG2/KD and HepG2/KD\# cells compared to that in the HepG2/NC cells at both the mRNA and protein levels (Fig. 3A and B). We also overexpressed USP39 in the HepG2 cells. USP39 expression was significantly increased in the HepG2/USP39 cells compared to that in the HepG2/vector cells at the protein level (Fig. 3C).

USP39 knockdown inhibits the growth of HepG2 cells and USP upregulation induces the opposite effects. To explore the function of USP39 on cell growth, HepG2 cells transfected with either the USP39-siRNA lentivirus or the NC lentivirus were monitored by Cellomics assay. HepG 2 cells containing USP39-siRNA and NC were seeded in 96-well plates, and cell growth was assayed every day for 5 days (Fig. 4A). The results showed that the cell growth was inhibited after downregulation of USP39 (Fig. 4B). MTT assay indicated that the growth of the HepG2 cells was significantly increased after USP39 was upregulated (Fig. 4C). Thus, these results indicated that USP39 knockdown suppressed the growth of HCC cells and USP39 overexpression had opposite effects.

USP39 induces G2/M arrest. USP39 was initially reported to regulate the cell cycle checkpoint (19-21). Moreover, we examined cell cycle change by FCM. We found that USP39 knockdown in the HepG2 cells showed a lower cell population in the G0/G1 stage and a higher cell population in the G2/M stage (Fig. 5A). HepG2/KD cells showed an increased apoptosis rate compared with the HepG2/NC cells (Fig. 5B).
Effect of USP39 on xenograft tumor growth. To investigate the effect of USP39 on xenograft tumor growth, we injected HepG2 cells containing the USP39 shRNA or the nonsilencing target RNA into nude mice. Immunohistochemical result showed that USP39 expression in the HepG2/ KD-xenografted tumors was significantly reduced compared to that in the xenografted tumors derived from HepG2/NC cells (Fig. 6C). Moreover, cell proliferation in the HepG2/KD-xenografted tumors was significantly decreased as demonstrated by anti-PCNA staining (Fig. 6C). Furthermore, USP39 knockdown significantly decreased xenografted tumor growth in the HepG2 cells (Fig. 6A and B). These results indicate that USP39 contributes to HCC tumor growth in vivo.

USP39-induced G2/M arrest depends on FoxM1. To further investigate the molecular mechanisms of USP39 on cell proliferation, we examined the expression of various important cell cycle proteins. We found that the level of p-Cdc2 in the HepG2/KD cells was upregulated while the levels of p-myt1 and p-Cdc25c were downregulated when compared to the levels in the HepG2/NC cells. Yet, the protein levels of total $\mathrm{Cdc} 2$, myt1 and $\mathrm{Cdc} 25 \mathrm{c}$ were not changed (Fig. 7A). When USP39 was upregulated in the HepG2 cells, the p-Cdc2 level was decreased compared with the level in the control cells (Fig. 7B). Additionally, p-Cdc2 was upregulated in the HepG2/KD-xenografted tumors (Fig. 6C). PLK1, the upstream factor of $\mathrm{Cdc} 2$, was decreased in the HepG2/KD cells compared with the HepG2/NC cells (Fig. 7C). Furthermore, FoxM1 which controls the level of PLK1, was decreased in the HepG2/ KD cells. Cyclin B1, another target gene of FoxM1, was also decreased in the HepG2/KD cells (Fig. 7C). 

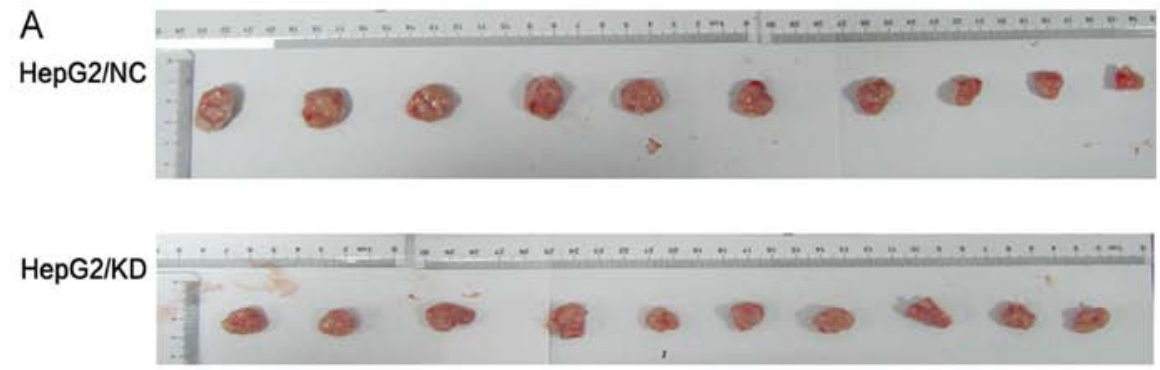

B

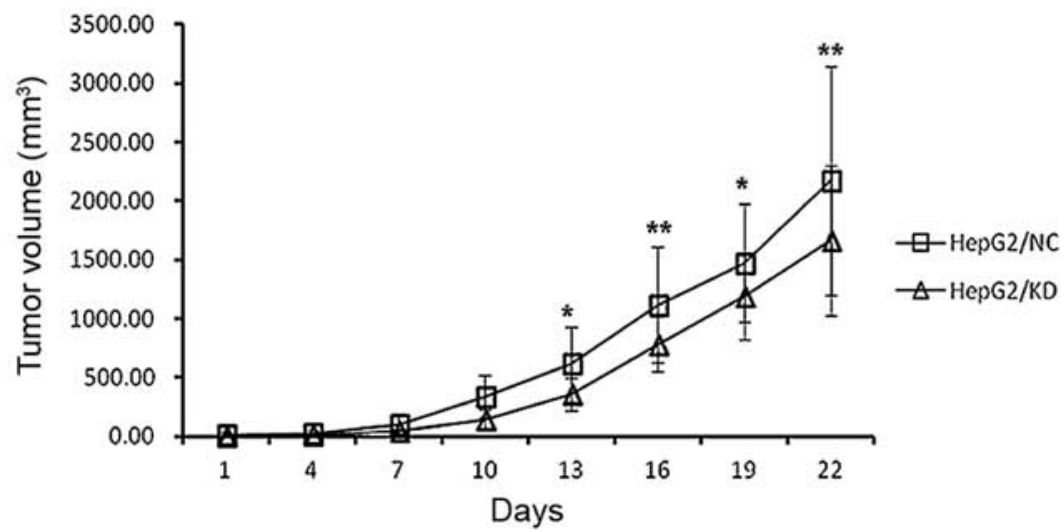

C

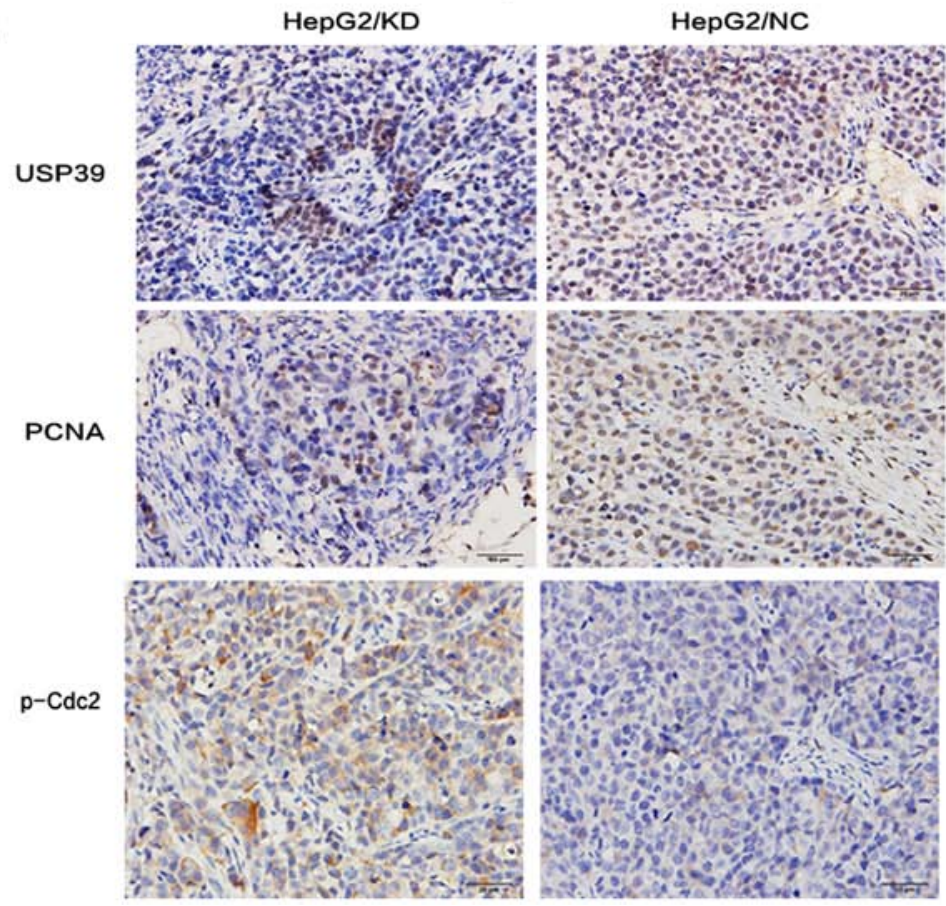

Figure 6. USP39 knockdown inhibits the growth of HepG2 cells in vivo. Scale bar, $20 \mu \mathrm{m}$; Values are expressed as the mean $\pm \mathrm{SE}, \mathrm{n}=10$; ${ }^{*} \mathrm{p}<0.05$, ${ }^{* *} \mathrm{p}<0.01$. (A) USP39 knockdown in HepG2 cells decreased xenografted tumor growth in nude mice. (B) The tumor growth was significantly inhibited. (C) USP39 knockdown in HepG2 cells (top panels) decreased the cell proliferation (middle panels) in the xenografted tumors.

\section{Discussion}

Since USP39 is an important factor for SR-related proteins targeting a set of key regulatory genes by modulating RNA splicing (23), this research aimed to discover the roles of USP39 in HCC. In the present study, we demonstrated for the first time that USP39 knockdown led to a defect in mRNA splicing and further caused cell cycle arrest in the G2/M phase in HCC. Firstly, we compared the expression of USP39 in tumor tissues and corresponding adjacent normal liver tissues from more than $100 \mathrm{HCC}$ patients. Notably, the expression of USP39 was associated with the pathological grade, suggesting that USP39 may play an important role in HCC.

To confirm the role of USP39 in HCC, we selected one effective shRNA sequence to study in the subsequent research, and we successfully established stable USP39knockdown and USP39-overexpressing HepG2 cells. We found that USP39 knockdown significantly inhibited the growth and colony formation of the HepG2 cells. Conversely, USP39 overexpression significantly enhanced the growth 


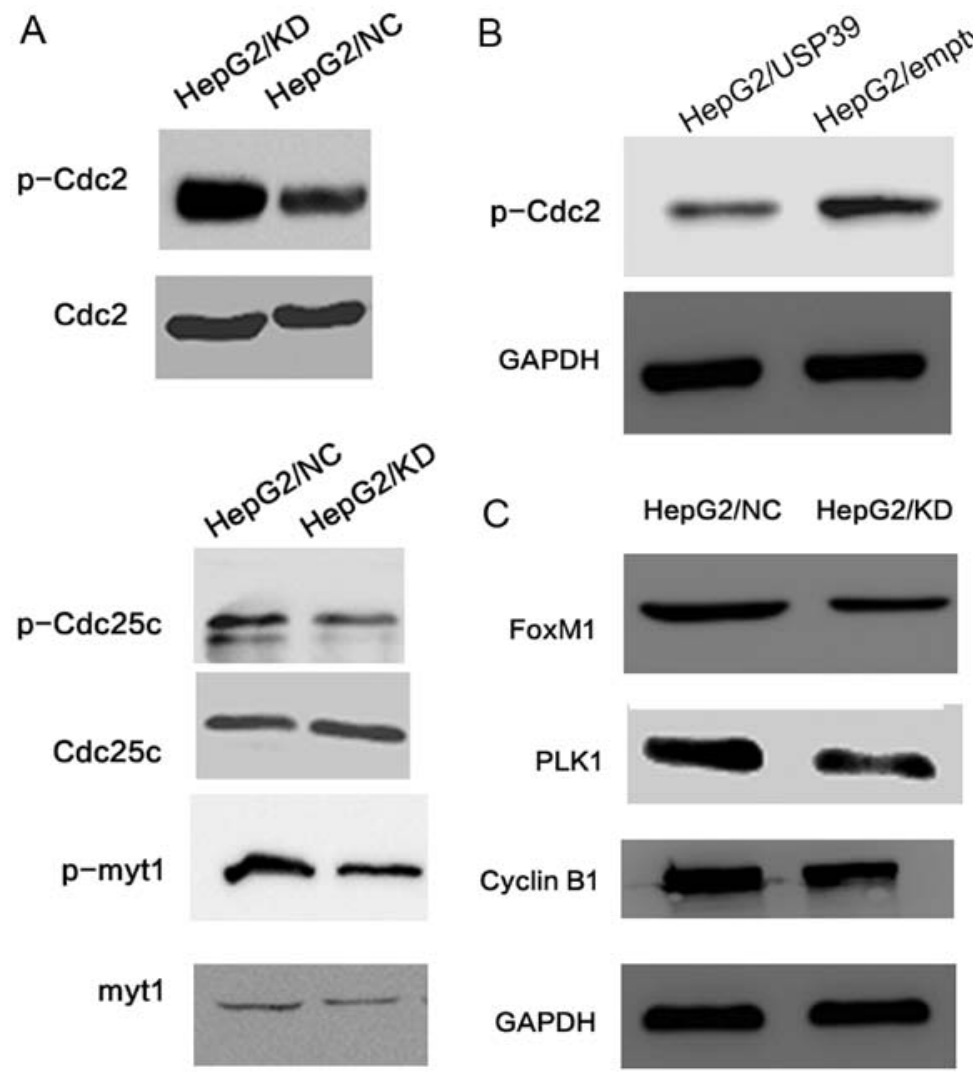

Figure 7. Mechanisms involved in the regulation of tumor growth by USP39. Western blotting was carried out to detect the expression of important cell cycle checkpoint proteins. (A) The level of p-Cdc2 was upregulated after USP39 knockdown, while the levels of p-Cdc25c and p-myt1 were downregulated by USP knockdown. The levels of $\mathrm{Cdc} 2, \mathrm{Cdc} 25 \mathrm{c}$ and myt1 were not changed. (B) The expression of p-Cdc2 was downregulated in the USP39-overexpressing tumor cells. (C) FoxM1, as well as its target gene PLK1 and cyclin B1, were decreased after USP39 knockdown in the HepG2 cells.

and colony formation of the HepG2 cells. Moreover, USP39 knockdown led to G2/M arrest and induced cell apoptosis in the HepG2 cells. These data indicate that USP39 knockdown inhibited HCC growth more likely through inducing G2/M phase arrest. Furthermore, the tumor growth was decreased in the USP39/KD-engrafted mice compared to the growth in the control mice. Thus, USP39 ablation inhibited the growth of HCC cells in vitro and in vivo.

Since sustained proliferative signaling is one of the hallmarks of cancer, inhibition of signaling is an effective approach in cancer therapy (24). Checkpoints of the cell cycle control the proper timing of cell cycle events by enforcing the dependency of late events on the completion of early events (25). Consequently, a checkpoint block can result in cell cycle arrest and significantly alter the activity of cell proliferation. A previous study revealed that downregulation of USP39 inactivated the G0/G1 arrest in the zebrafish in vivo by splicing the rb1 that plays an important role in the transition from G0 to G1 phase (20). A similar effect was observed in human breast cancer in vitro (21). Nevertheless, our research showed that USP39 knockdown induced significant $\mathrm{G} 2 / \mathrm{M}$ arrest in the $\mathrm{HCC}$ cell lines, along with the suppression of Cdc2 activity (the level of p-Cdc2 at Tyr15 site upregulation). We also verified a similar result of $\mathrm{p}-\mathrm{Cdc} 2$ expression in the xenograft tumors obtained from the nude mice as that in vitro. It is well-known that the G2/M transition is regulated by a complex of $\mathrm{Cdc} 2$ and cyclin $\mathrm{B} 1$, and a decrease in $\mathrm{Cdc} 2$ activity ultimately leads to G2/M arrest $(26,27)$.
There are three major phosphorylation sites (Thr161, Tyr15 and Thr14) by which Cdc2 kinase activity is regulated (28). Phosphorylation at Thr14 and Tyr15 is carried out by Weel and Myt1 protein kinases and results in the inhibition of Cdc2 (29-31). PLK1 starts the mitotic cascade by phosphorylating and activating $\mathrm{Cdc} 25 \mathrm{C}$ phosphatase and inhibits myt1 activity, which in turn establishes a feedback amplification loop that influences the cell cycle (32). A previous study demonstrated that $\mathrm{Cdc} 2$ is activated by the dephosphorylation at Tyr15. In the present study, we found that USP39 knockdown induced G2/M arrest in HepG2 cells, and increased the expression of p-Cdc2 (Thr15). The expression of PLK1 was decreased after USP39 knockdown. FoxM1 was reported to regulate expression of PLK1 (33) and to undergo alternative splicing (8). Moreover, expression of FoxM1 was also decreased after USP39 knockdown in the HepG2 cells. Cyclin B1, another target gene of FoxM1, was also decreased following USP39 knockdown. van Leuken et al found that USP39 could control the mRNA level of Aurora B, and Aurora B is also a target gene of FoxM1 (19). Thus, we can tentatively conclude that USP39 may contribute to the mRNA splicing of FoxM1.

In summary, by using in vitro and in vivo approaches, we provide evidence that USP39 knockdown inhibited the tumor growth in human $\mathrm{HCC}$ by inducing G2/M arrest, at least partly, due to FoxM1 splicing. USP39 expression was associated with the pathological tumor stage of HCC, suggesting that USP39 may be considered as a promising molecular target for HCC. 


\section{Acknowledgements}

This study was supported by grants from the National Natural Science Foundation of China (no. 31300103), the Clinical Medical Center for Hepatobiliary Disease of Jiangsu Province (no. ZX201105) and the Clinical Medical Center for Digestive Disease of Jiangsu Province (no. BL2012001). We thank Drs Huiping Yu and Jun Yang from the Department of Pathology, The Affiliated Drum Tower Hospital, School of Medicine, Nanjing University, for their assistance in the analysis for IHC staining.

\section{References}

1. Villanueva A, Minguez B, Forner A, Reig M and Llovet JM: Hepatocellular carcinoma: Novel molecular approaches for diagnosis, prognosis, and therapy. Annu Rev Med 61: 317-328, 2010.

2. Huynh H: Molecularly targeted therapy in hepatocellular carcinoma. Biochem Pharmacol 80: 550-560, 2010.

3. Fisher D, KrasinskaL, Coudreuse D and Novák B: Phosphorylation network dynamics in the control of cell cycle transitions. J Cell Sci 125: 4703-4711, 2012.

4. Nurse P: A long twentieth century of the cell cycle and beyond Cell 100: 71-78, 2000.

5. Martin SJ, McGahon AJ, Nishioka WK, LaFace D, Guo X, Th'ng J, Bradbury EM and Green DR: p34cdc2 and apoptosis. Science 269: 106-107, 1995.

6. Laoukili J, Stahl M and Medema RH: FoxM1: At the crossroads of ageing and cancer. Biochim Biophys Acta 1775: 92-102, 2007.

7. Koo CY, Muir KW and Lam EW: FOXM1: From cancer initiation to progression and treatment. Biochim Biophys Acta 1819: 28-37, 2012.

8. Ye H, Kelly TF, Samadani U, Lim L, Rubio S, Overdier DG, Roebuck KA and Costa RH: Hepatocyte nuclear factor 3/fork head homolog 11 is expressed in proliferating epithelial and mesenchymal cells of embryonic and adult tissues. Mol Cell Biol 17: 1626-1641, 1997.

9. Wang IC, Chen YJ, Hughes D, Petrovic V, Major ML, Park HJ, Tan Y, Ackerson T and Costa RH: Forkhead box M1 regulates the transcriptional network of genes essential for mitotic progression and genes encoding the SCF (Skp2-Cks1) ubiquitin ligase. Mol Cell Biol 25: 10875-10894, 2005.

10. Costa RH: FoxM1 dances with mitosis. Nat Cell Biol 7: 108-110, 2005.

11. Nagata A, Igarashi M, Jinno S, Suto K and Okayama H: An additional homolog of the fission yeast $\operatorname{cdc} 25^{+}$gene occurs in humans and is highly expressed in some cancer cells. New Biol 3: 959-968, 1991.

12. Galaktionov K and Beach D: Specific activation of cdc 25 tyrosine phosphatases by B-type cyclins: Evidence for multiple roles of mitotic cyclins. Cell 67: 1181-1194, 1991.

13. Molinari M, Mercurio C, Dominguez J, Goubin F and Draetta GF: Human Cdc25 A inactivation in response to $S$ phase inhibition and its role in preventing premature mitosis. EMBO Rep 1: 71-79, 2000.

14. Karlsson C, Katich S, Hagting A, Hoffmann I and Pines J: Cdc25B and Cdc25C differ markedly in their properties as initiators of mitosis. J Cell Biol 146: 573-584, 1999.
15. De Souza CP, Ellem KA and Gabrielli BG: Centrosomal and cytoplasmic Cdc2/cyclin B1 activation precedes nuclear mitotic events. Exp Cell Res 257: 11-21, 2000.

16. Hoffmann I, Clarke PR, Marcote MJ, Karsenti E and Draetta G: Phosphorylation and activation of human cde25-C by cdc2cyclin B and its involvement in the self-amplification of MPF at mitosis. EMBO J 12: 53-63, 1993.

17. Perdiguero E and Nebreda AR: Regulation of Cdc25C activity during the meiotic G2/M transition. Cell Cycle 3: 733-737, 2004.

18. Reyes-Turcu FE, Ventii KH and Wilkinson KD: Regulation and cellular roles of ubiquitin-specific deubiquitinating enzymes. Annu Rev Biochem 78: 363-397, 2009.

19. van Leuken RJ, Luna-Vargas MP, Sixma TK, Wolthuis RM and Medema RH: Usp39 is essential for mitotic spindle checkpoint integrity and controls mRNA-levels of aurora B. Cell Cycle 7: 2710-2719, 2008.

20. Ríos Y, Melmed S, Lin S and Liu NA: Zebrafish usp39 mutation leads to rb1 mRNA splicing defect and pituitary lineage expansion. PLoS Genet 7: e1001271, 2011.

21. Wang H, Ji X, Liu X, Yao R, Chi J, Liu S, Wang Y, Cao W and Zhou Q: Lentivirus-mediated inhibition of USP39 suppresses the growth of breast cancer cells in vitro. Oncol Rep 30: 2871-2877, 2013.

22. Sun XT, Yuan XW, Zhu HT, Deng ZM, Yu DC, Zhou X and Ding YT: Endothelial precursor cells promote angiogenesis in hepatocellular carcinoma. World J Gastroenterol 18: 4925-4933, 2012.

23. Makarova OV, Makarov EM and Lührmann R: The 65 and $110 \mathrm{kDa}$ SR-related proteins of the U4/U6.U5 tri-snRNP are essential for the assembly of mature spliceosomes. EMBO J 20: 2553-2563, 2001.

24. Hanahan D and Weinberg RA: Hallmarks of cancer: The next generation. Cell 144: 646-674, 2011.

25. Lapenna $S$ and Giordano A: Cell cycle kinases as therapeutic targets for cancer. Nat Rev Drug Discov 8: 547-566, 2009.

26. Smits VA, Klompmaker R, Vallenius T, Rijksen G, Mäkela TP and Medema RH: p21 inhibits Thr161 phosphorylation of Cdc2 to enforce the G2 DNA damage checkpoint. J Biol Chem 275: 30638-30643, 2000

27. Guadagno TM and Newport JW: Cdk2 kinase is required for entry into mitosis as a positive regulator of $\mathrm{Cdc} 2$-cyclin $\mathrm{B}$ kinase activity. Cell 84: 73-82, 1996.

28. Atherton-Fessler S, Liu F, Gabrielli B, Lee MS, Peng CY and Piwnica-Worms H: Cell cycle regulation of the p34cdc2 inhibitory kinases. Mol Biol Cell 5: 989-1001, 1994.

29. Norbury C, Blow J and Nurse P: Regulatory phosphorylation of the p34cdc2 protein kinase in vertebrates. EMBO J 10: 3321-3329, 1991.

30. McGowan $\mathrm{CH}$ and Russell P: Human Weel kinase inhibits cell division by phosphorylating p34cdc2 exclusively on Tyr15. EMBO J 12: 75-85, 1993.

31. Wells NJ, Watanabe N, Tokusumi T, Jiang W, Verdecia MA and Hunter T: The C-terminal domain of the Cdc2 inhibitory kinase Myt1 interacts with Cdc2 complexes and is required for inhibition of G(2)/M progression. J Cell Sci 112: 3361-3371, 1999.

32. Cogswell JP, Brown CE, Bisi JE and Neill SD: Dominant-negative polo-like kinase 1 induces mitotic catastrophe independent of cdc25C function. Cell Growth Differ 11: 615-623, 2000.

33. Dibb M, Han N, Choudhury J, Hayes S, Valentine H, West C, Ang YS and Sharrocks AD: The FOXM1-PLK1 axis is commonly upregulated in oesophageal adenocarcinoma. Br J Cancer 107: 1766-1775, 2012 